\title{
A Study of Agricultural Meteorological Monitoring System Based on Wireless Sensor Networks
}

\author{
Li Ma $^{1,2,3}$, Jingzhou Yan ${ }^{1,2}$, Shuangshuang Yan ${ }^{1,2}$, Baowei Wang ${ }^{1,2}$, Kuo Liao ${ }^{3,4}$ and \\ Jin Wang ${ }^{1,2}$ \\ ${ }^{1}$ Jiangsu Engineering Center of Network Monitoring, Nanjing University of \\ Information Science and Technology, Nanjing 210044 \\ ${ }^{2}$ School of Computer and Software, Nanjing University of Information, \\ Science and Technology, Nanjing 210044 \\ ${ }^{3}$ Key Laboratory of Meteorological Disaster of Ministry of Education, Nanjing \\ University of Information Science and Technology, Nanjing 210044 \\ ${ }^{4}$ Ningde Meteorological Burean, Ningde 352100
}

\begin{abstract}
In order to keep the sustainable development of agricultural industry, monitoring the four basic essential elements' changes, which are temperature, humidity, light intensity, carbon dioxide, and applying the corresponding solution is really necessary. An agriculture meteorological monitoring system bith ZigBee wireless sensor networks (WSNs) and wireless communication technology is designed in this paper. Sensor nodes are distributed in the measurement area and form a self-organizing network. The sink collects information which transmitted from sensor hodes and then, sends the information to the server. Server will summarize the information then taking aralysis and processing. At the end, the server will give some corresponding warning and forecast. The cost of this system is low and could improve the accuract and timetiresy in agricultural meteorological monitoring.
\end{abstract}

Key Words : ZigBee, WSN,Agricultural Meteorological Monitoring

\section{Introduction}

Crops growth and yield is often affected by some meteorological factors [1]. Therefore, it is necessary to strengthen the essential elements monitoring in agricultural industry. Essential elements contain temperature, humidity, light intensity and carbon dioxide. With the development of wireless sensor networks [2-6] and requirements of agricultural meterological monitoring, an agricultural meteorological monitoring system with wireless sensor networks is designed to realize the intelligent agriculture monitoring. The system can predict environmental trends and the corresponding impact on agriculture. By collecting the information like this, people can take appropriate measures to ensure stable and high yield of crops. The system could improve accuracy and timeliness in agricultural meteorological monitoring.

Cluster network is selected since it has simple construction, with multi-function and good fault-tolerance. When one or more nodes are damaged, the network could form a self-organizing network again and still functional.

The system combines ZigBee wireless sensor networks with wireless communication technology, sends parameters to mobile phone users through GSM network. The system not only can provide monitoring and warning, but also can give another way to provide intelligent 
prediction so that the user can be able to do the remote monitoring. The architecture of system is illustrated in Figure 1.

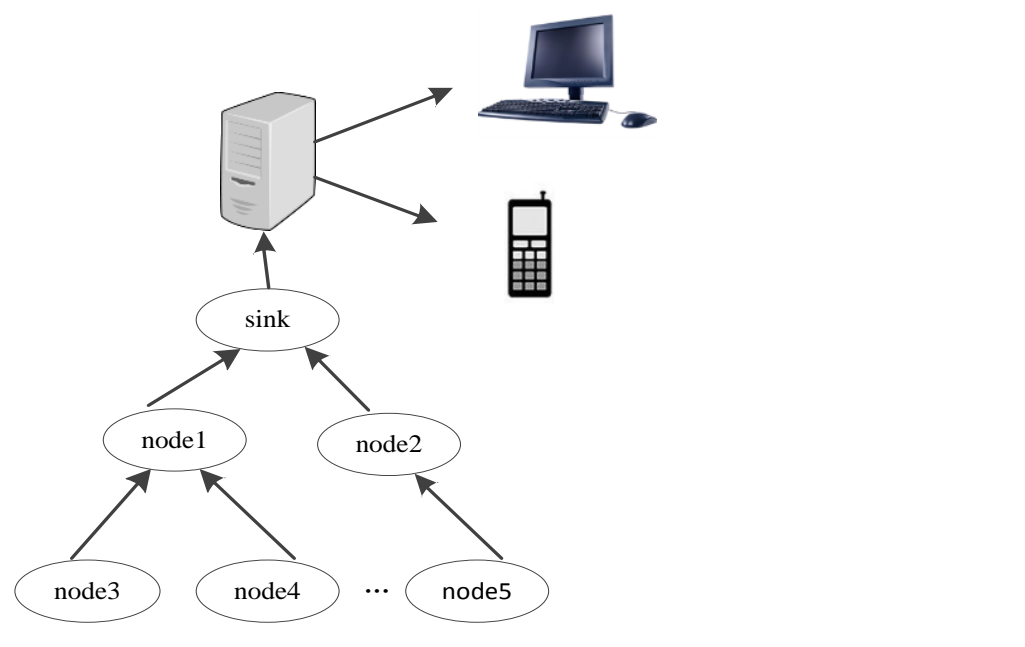

Figure 1. Architecture of Agricultural Meteorological Monitoring System

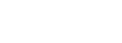

The system provides two kinds of monitoring schemes. The first scenario designed a software application to display the monitoring interface on computer. The sink collects information which transmitted from sensor nodes and then sends the information to the server. User could check each node's situation through the software. The second scenario uses mobile phone text to transmit the warning. Computer will summarize the information, then taking analysis and processing. Once it is beyond the threshold, the system will send mobile phone text to inform the user.

\section{Design and Imprementation}

In our system, the nodes collect temperature, humidity and light intensity information. We use DHT11 to collect temperature and humidity information and ON9658F collect light intensity information C 2430 is used as the processor module and communication module of the nodes and proxides the power that the system need through the battery. The structure of nodes is illustrated in Figure 2.

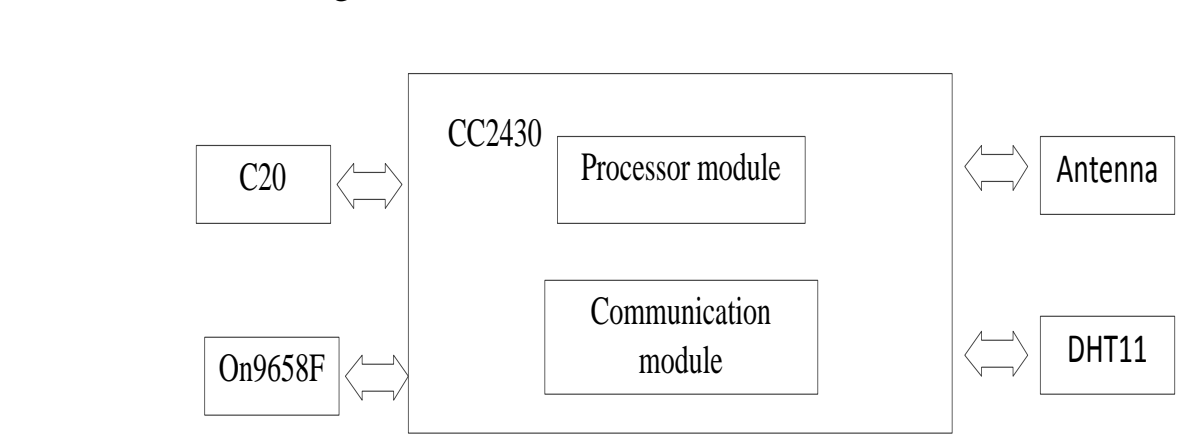

Figure 2. The Structure of Nodes 


\subsection{Some Work in Hardware}

(1) The processor

The CC2430 is a true System-on-Chip (SoC) solution specifically tailored for IEEE 802.15.4 and ZigBee ${ }^{\mathrm{TM}}$ applications. The CC2430 combines the excellent performance of the leading CC2420 RF transceiver with an industry-standard enhanced $8051 \mathrm{MCU}, 32 / 64 / 128$ KB flash memory, 8 KB RAM and many other powerful features [7]. The CC2430 has high performance, low power and have well anti-noise performance. We can use few external components to build ZigBee nodes.

The sensor nodes were composed of CC2430 and other external circuits such as crystal oscillator, bias resistance and coupling capacitance. Barron circuit is used as the node's antenna part, Barron circuit were composed of discrete inductors and capacitance. Using two capacitance and a crystal oscillator which in $32 \mathrm{MHz}$ to make up the clock circuit. Three lights are designed to indicate the working status of the node power supply status, hetworking status and communication status. The schematic diagram of CC2430 is illustrated in Figure 3.

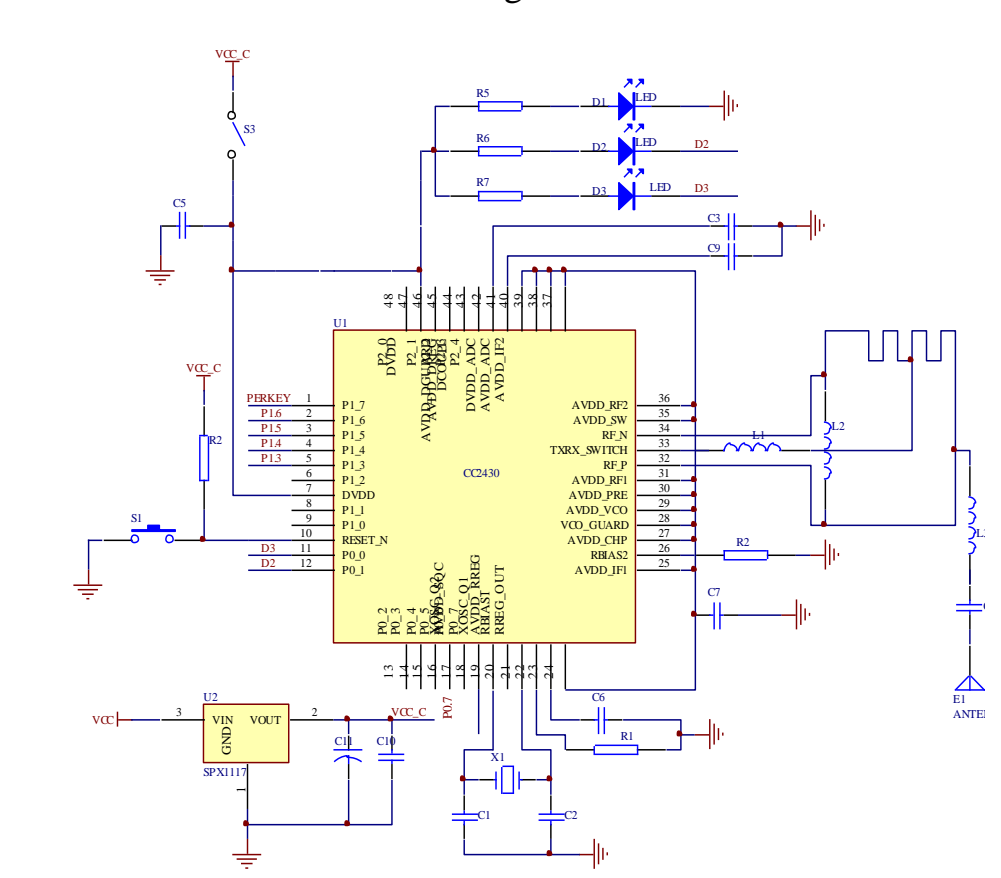

Figure 3. The Schematic Diagram of CC2430

(2) Kight sensor part

On $9658 \mathrm{~F}$ is used here to collect light intensity information. The On9658F is a photoelectrical integrated optical sensor, with double sensitive receiver, and add visible light pass and N-IR cut optical filters. It is highly sensitive to visible light, and varies linearly with illumination changing [8]. The On9658F is widely used in digital product and industry device. We know the voltage is proportional to current, and the current is varies linearly with illumination changing. So the voltage also with illumination is changing. We use the transformation of voltage to calculate the value of light intensity. The schematic diagram of On9658F is illustrated in Figure 4. 


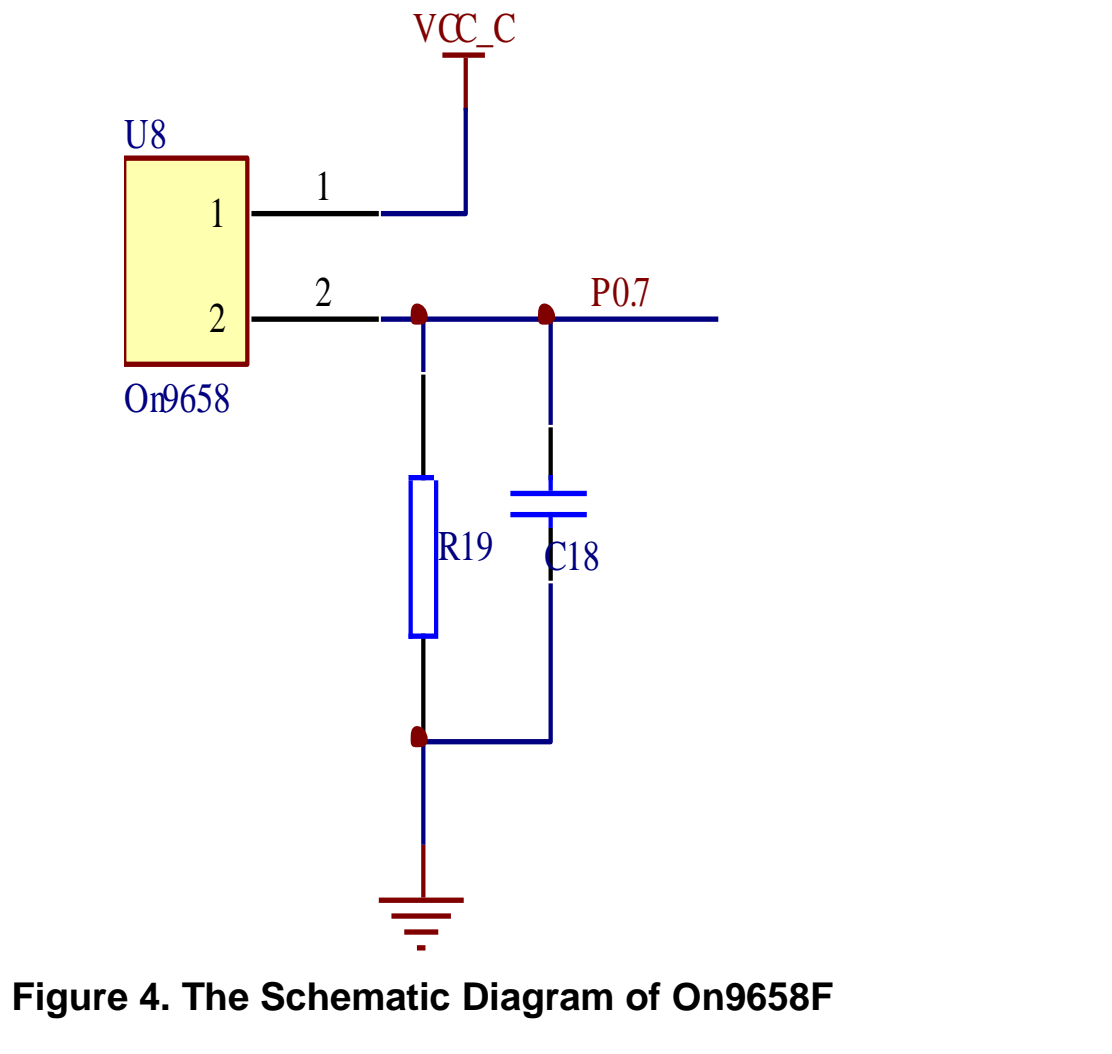

In CC2430, the ADC supports up to 14-bitanalog-to-digital conversion. ADC is used to process voltage value which wals send by $0 \mathrm{n} 9658 \mathrm{~F}$. In the end, the actual light intensity value was calculated.

\section{(3) Temperature and Humidity Sensor}

DHT11 temperature and hamidity sensor is a complexed temperature and humidity sensor which contains a calibrated digital signal output. Its professional digital module technology ensures the high reliabitity and excellent long-term stability[9].DHT11 was widely used for its small size, low power dissipation and strong anti-interference. DHT11 includes a resistive element and a NTO wet and temperature measuring devices to collect temperature and humidity infornhation.

DHT11 ases 1-Wire Bus technology. In other words, DHT11 only need one cable to finish data transmission and sensor control. After power it on,DHT11 need 1s to complete the initialization. At the same time, DHT11 will collect and record temperature and humidity data. This time, the pins of DHT11 is at the state of input. When we give DHT11 a low level signals that is greater than $18 \mathrm{~ms}$, DHT11 will get into the output state. Then,DHT11 sends 80 us high level to the processor and processor responds with 80 us high level to DHT11, signing the preparation is ready. After that, data will be transmitted from the DHT11 to the processor and DHT11 will get into another turn of data transmittion

In this system, DHT11 is used to collect temperature and humidity information. The first pins is connected with working voltage, second pins connects with data communication and fourth pins connects with grounding. The schematic diagram of DHT11 is illustrated in Figure 5. 


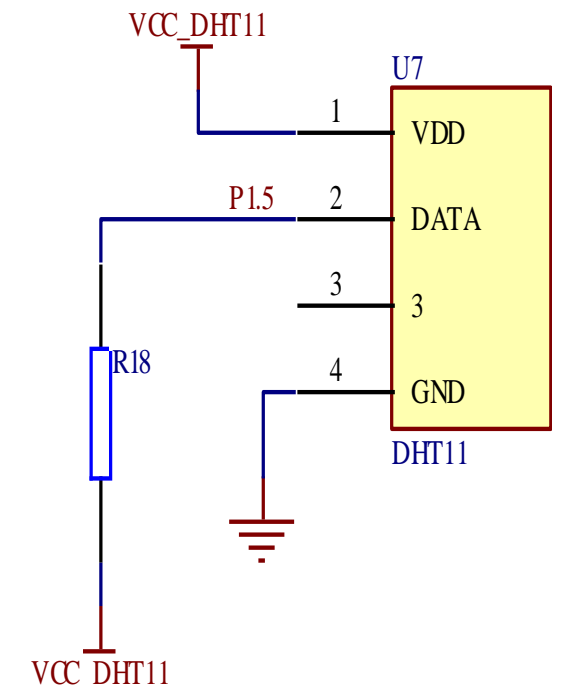

Figure 5. The Schematic Diagram of DHT11

(4) Carbon Dioxide Sensor

In this system, C20 is used to collect ${ }^{\circ}$ cafon dioxide information. $\mathrm{C} 20$ is made by by company called GSS. C20 was used in system for its small size and strong anti-interference.It could be well used in agriculture meteorological monitonng. The second pins is used to send data and third pins uesd to receive datarthe schenatic diagram of C20 is illustrated in Figure 6.

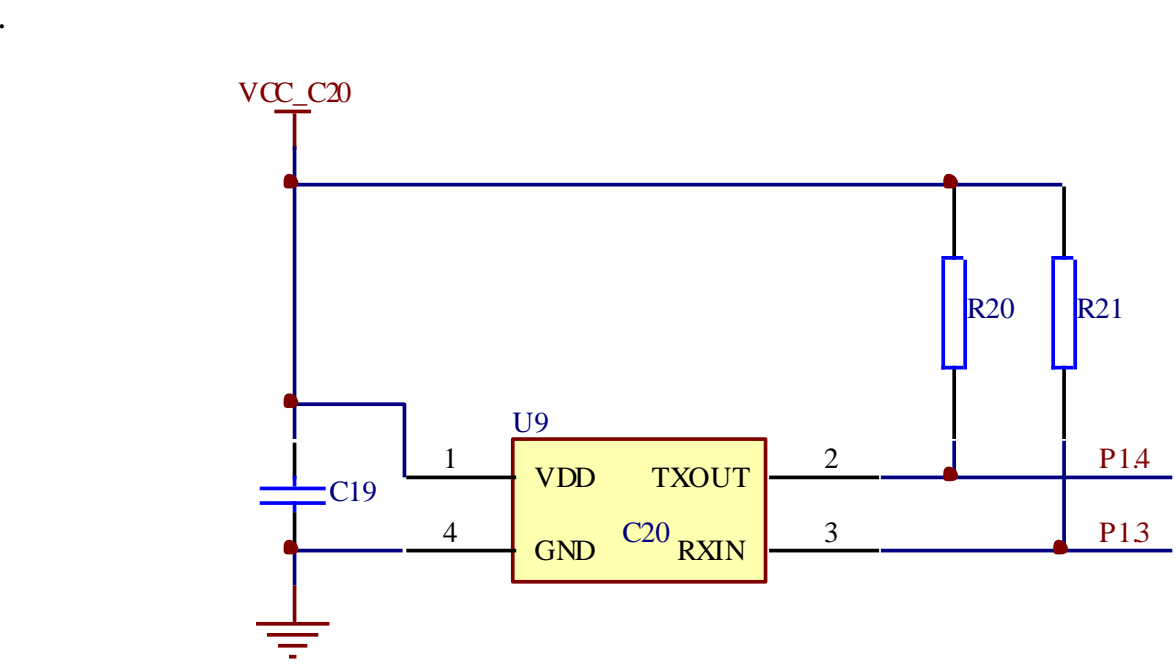

Figure 6. The Schematic Diagram of $\mathrm{C20}$

\subsection{Some Work in Procedure}

The system adopted cluster network. Routing nodes and common nodes are set according to certain proportion. Each node has different task.

(1)The procedure design of sink

The task of the sink is completing the form of the network, searching for a new node, collecting node data from the net and sending them to the server. The procedure design of sink is illustrated in Figure 7. 


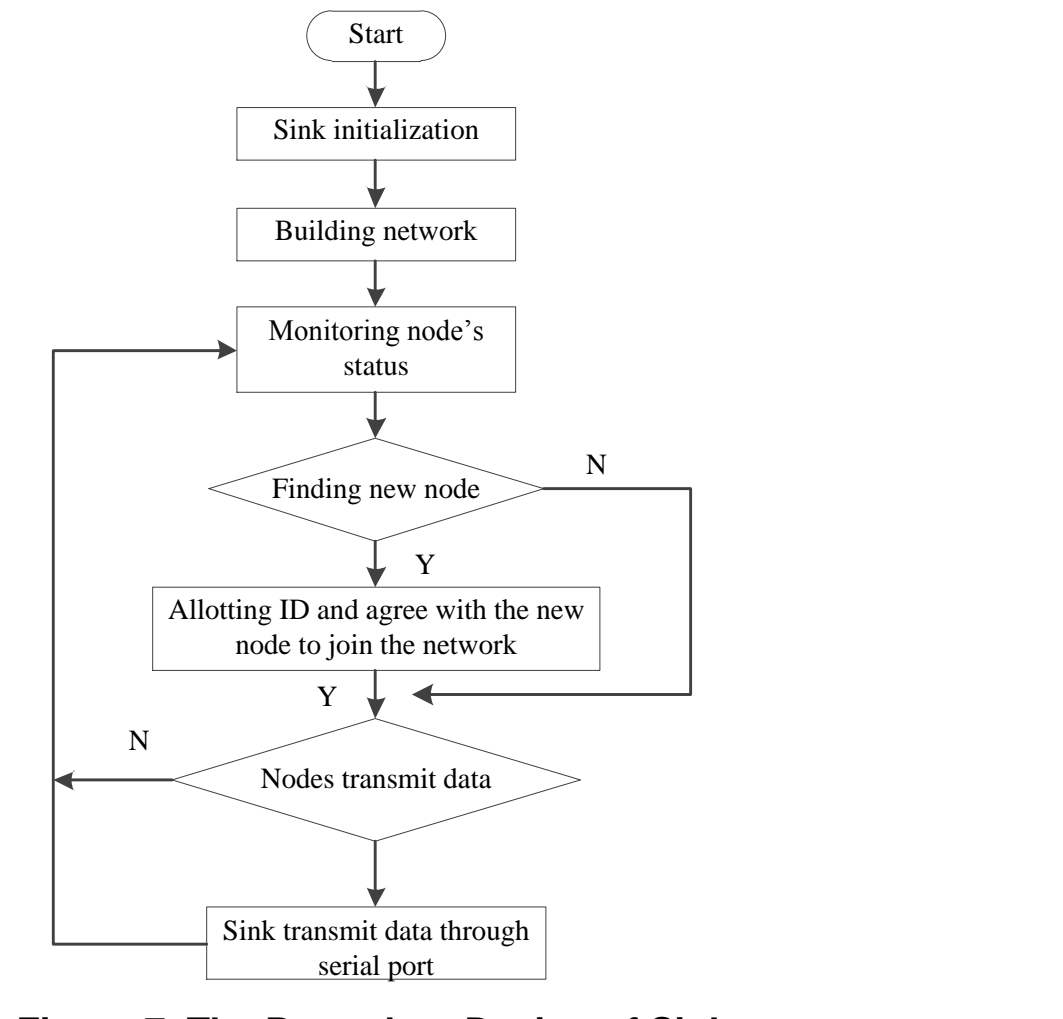

Figure 7. The Procedure Design of Sink

At the beginning, sink need to have an initialization. After initialization, the sink will monitor the nodes' status. If the sink find a new node, after checking whether it is connected, if not, it will allot ID and agree with the new node to join in the network. After that, the nodes will transmit data to the sink. In the end, the sink will transmit data through serial port.

\section{(2)The procedure design of oømmon nodes and routing nodes}

In this system, cluster network is applied. So, routing nodes and common nodes will be set according to a certain proportion. In order to prevent a node can't transmit the data when its routing node have blockage or damage, the terminal nodes will contain the search function. If a routing node is damaged or stop working, its common nodes will search a new routing node.

The design procedure of common node is illustrated in Figure 8(a). The nodes will start with an mitialization. After the initialization is done, the node will search the network and send a request to join in the network. Then, the node will give a low level signal no less than $18 \mathrm{~ms}$ to DHT11. After this, DHT11 will transfer into output state and send signal to CC2430. After accepting the data from DHT11, the node will receive voltage from On9658F and save the data after an analog-to-digital operation. In the end, Node will transmit data which it received and get into the next round of cycle.

For routing nodes in WSNs, it has similar process with common nodes. The difference is that it not only collects its own data but also collects other common node's data. At last, the nodes send the data to the sink. The design procedure of routing node is illustrated in Figure $8(b)$. 


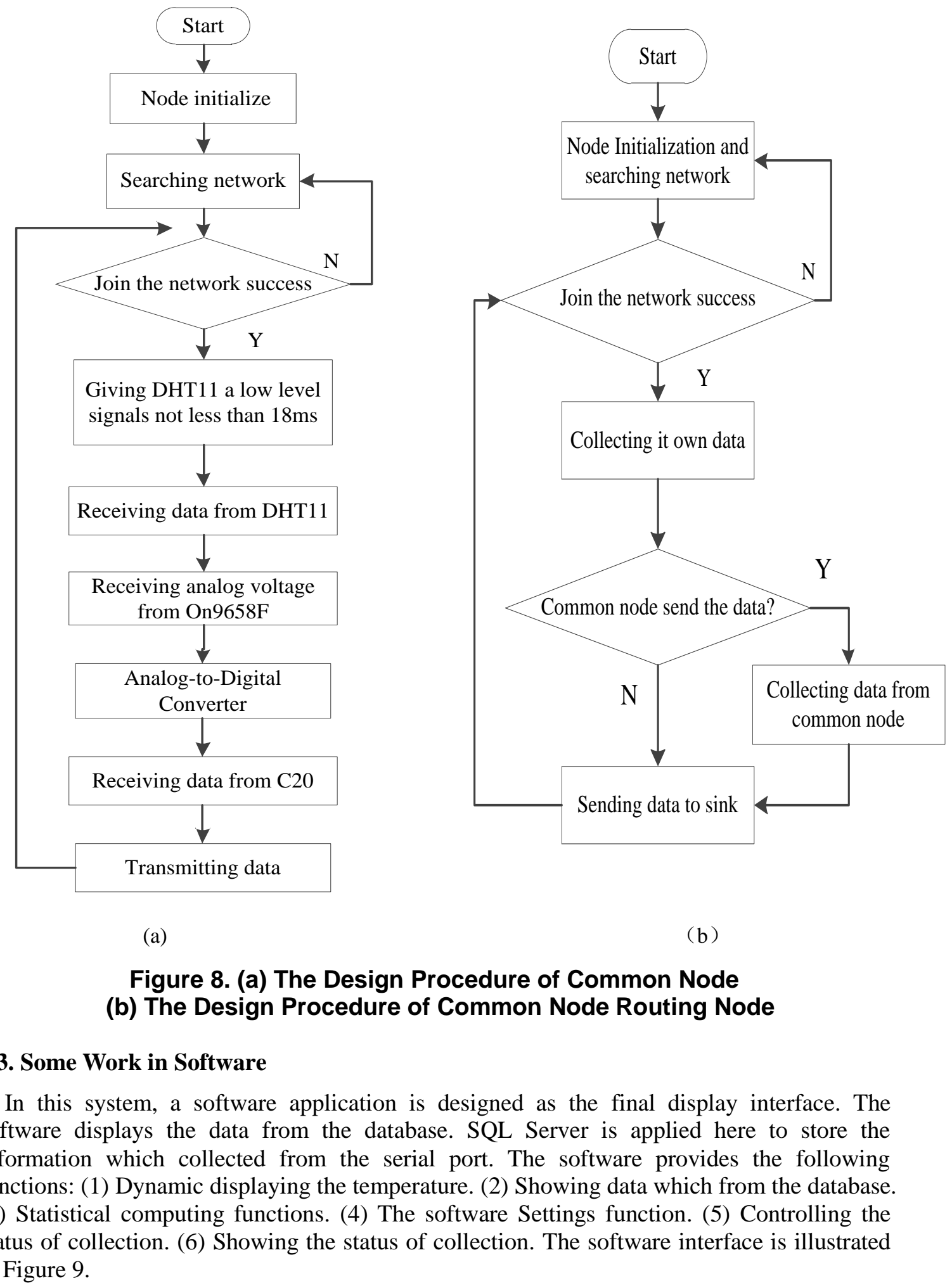




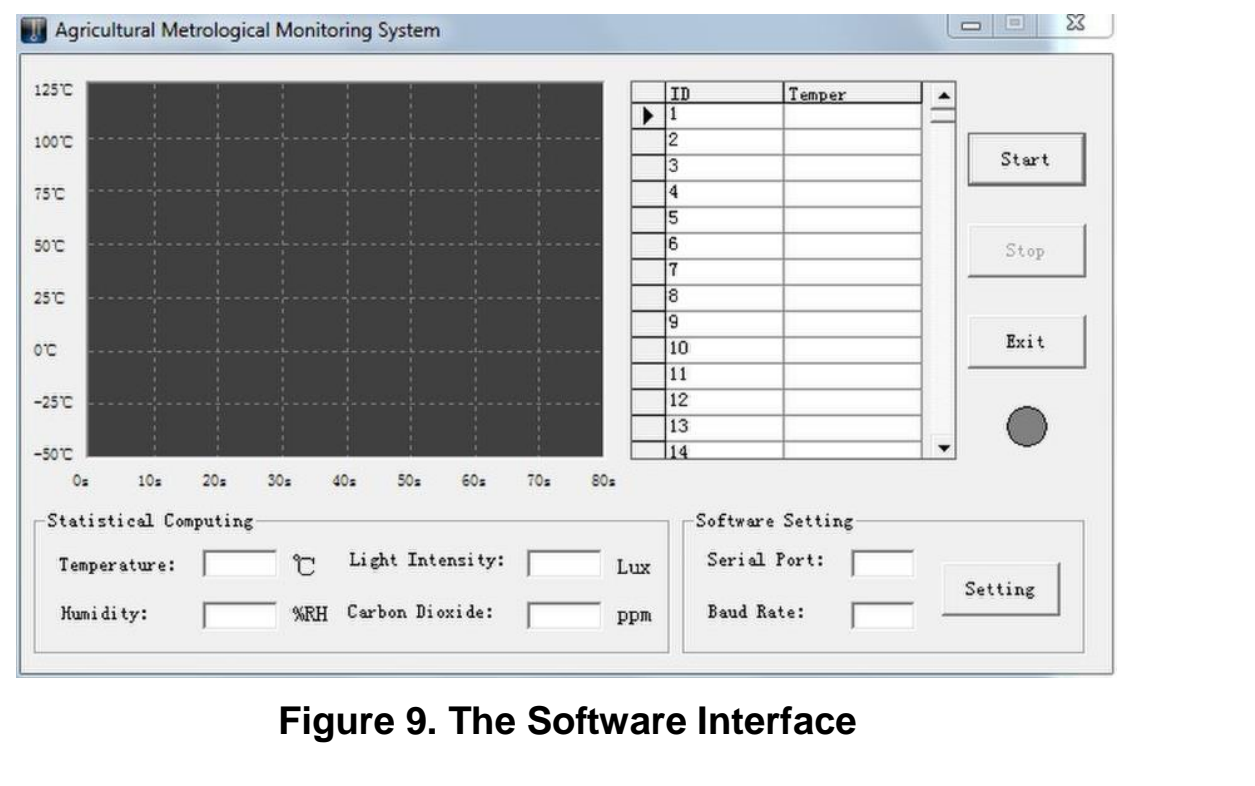

In the system, computer will summarize, the information, then taking analysis and processing. Once beyond the threshold, the system will use mobile phone text to tell the user. The software design is illustrated in Figure 10.

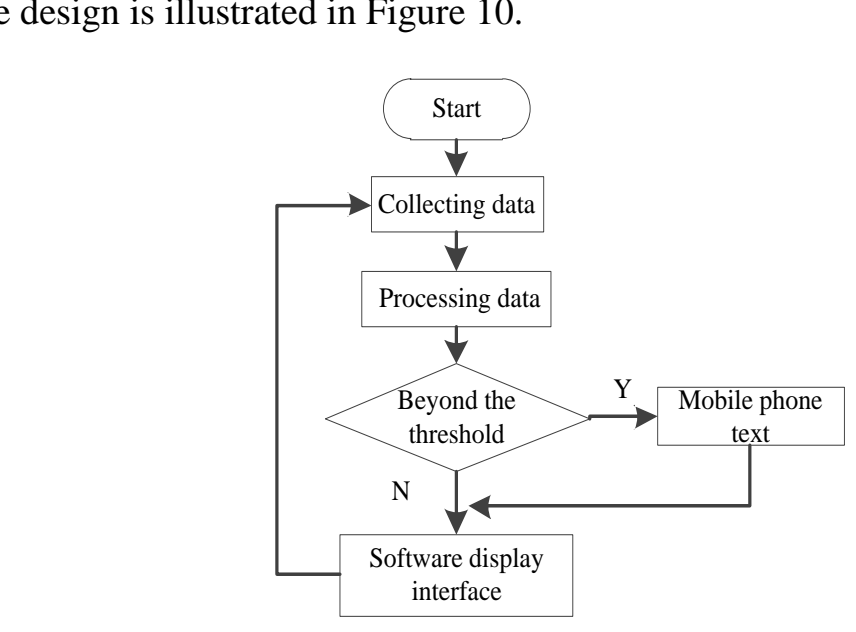

Figure 10. The Software Design

\section{Experiment and Results}

We deployed some nodes to validate the effectiveness of this system. The area of the test site is approximately 100 square meters. Set the sink as the center of the circle, 10 nodes (contain two routing nodes) are set around it. The distance between the each nodes is about 10 meters. After 20 hours, the experimenters found that the data packet transmitted stably and packet loss probability was low. The packet loss probability of nodes was between $2.07 \%$ and 7.08\%.The packet loss probability of nodes is illustrated in Figure 11. 


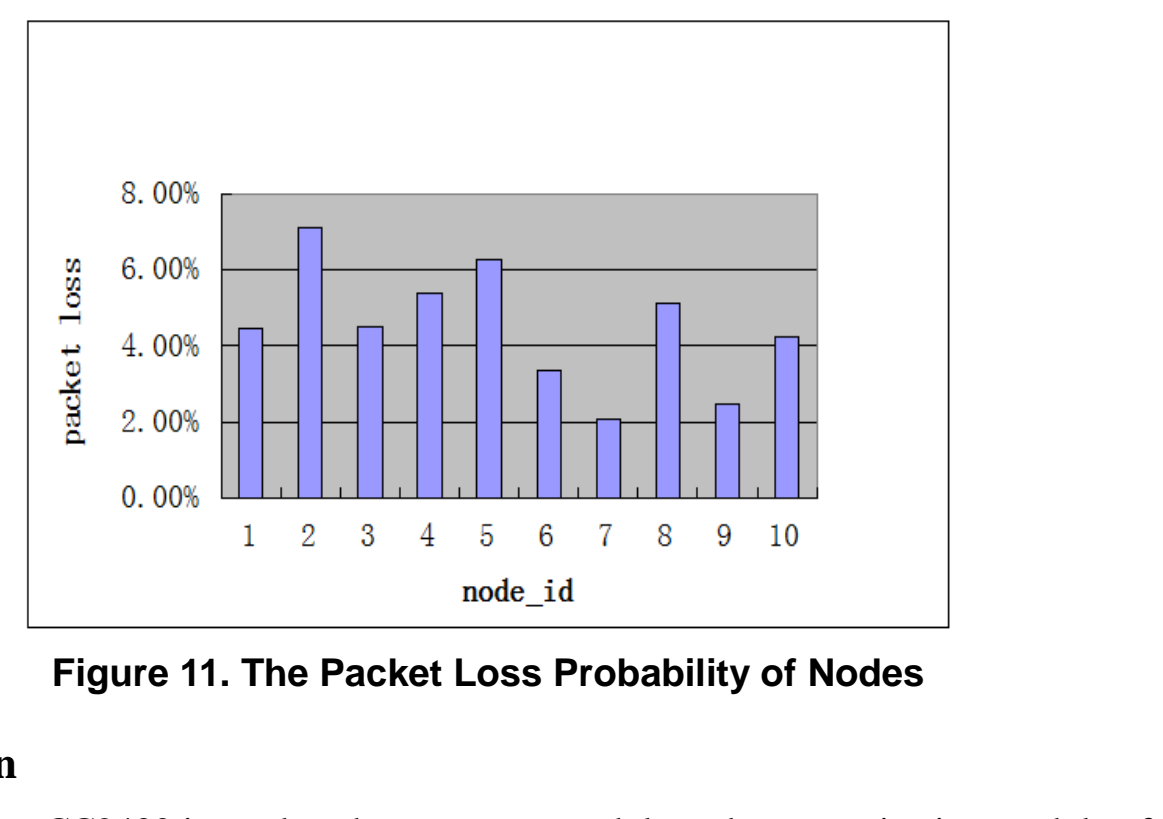

\section{Discussion}

In this system, CC2430 is used as the processor module and communication module of the nodes. The experimenters developed the nodes in the farmland and used DHT11 to collect temperature and humidity information, $0 \mathrm{n} 958 \mathrm{~F}$ is use to collect light intensity information and C20 is used to collected carbon dioxide information. Collected information will be sent to the routing nodes first. And then, will be sent to the sink. At last, the information will be sent to the server. If the server received the information, SQL Server will store the information which collected from the serial port. The software could show the data from the database. In this design, accurately data can be aequired with low cost. Applying WSNs to the meteorological monitoring is an inevitable trend. With the development of micro-electronics, computer and wireless communication technique, WSNs will have much more application prospects. The agricultural meteorological monitoring system based on wireless sensor networks will be applied widely.

\section{Acknowledgments}

Thanks for Zhen Yu Han, Bo Li and Lei Gu and also acknowledge the support of Jiangsu Engineering Center of Network Monitoring. This work is supported by the NSFC (61232016, 61173141, 61173142, 61173136, 61103215, 61373132, 61373133), GYHY201206033, 201301030 2013DFG12860, BC2013012 and PAPD fund. It was also supported by the Project of six personnel in Jiangsu Province (2013-WLW-012). It is also supported by the Natural science Foundation of Jiangsu Province (No. BK2012461) and by the Project of six personnel in Jiangsu Province (2013-WLW-012).

\section{References}

[1] J. P. Guo and S. H. Gao, "The experimental study on impacts of high temperature and high CO2 concentration on crops", Chinese Journal of Eco-Agriculture, vol. 10, no. 1, (2002).

[2] F. Y. Ren, H. N. Huang and C. Lin, "Wireless sensor networks", Journal of software, vol. 14, no. 17, (2003).

[3] J. Bruck, J. Gao and A. Jiang, "MAP: Medial axis based geometric routing in sensor networks", Wireless Networks, vol. 13, no. 6, (2007).

[4] J. M. Kahn, R. H. Katz and K. S. Pister, "Next century challenges: mobile networking for "Smart Dust"”, Proceedings of the 5th annual ACM/IEEE international conference on Mobile computing and networking, (1999), New York, USA. 
[5] Y. Liu, Y. He, M. Li, J. Wang, K. Liu, L. Mo, W. Dong, Z. Yang, M. Xi and J. Zhao, "Does wireless sensor network scale?", A measurement study on GreenOrbs, INFOCOM, 2011 Proceedings IEEE, (2011) April 10-15, Shanghai, China.

[6] G. Anastasi, A. Falchi, A. Passarella, M. Conti and E. Gregori, "Performance measurements of motes sensor networks", Proceedings of the 7th ACM international symposium on Modeling, analysis and simulation of wireless and mobile systems, (2004), New York, USA.

[7] G. S. Liang, D. J. Liu and F. Z. Hao, "Design of ZigBee wireless network node based on CC2430", Electronic Design Engineering, vol. 18, no. 2, (2010).

[8] M. Zengwei, M. Jinru and L. Yamin, "Intelligent Greenhouse Monitoring and Control System Design Based on Wireless Fidelity", Journal of Agricultural Mechanization Research, vol. 2, (2011).

[9] N. Tianlong, "Application of Single Bus Sensor DHT11 in Temperature Humidity Measure and Control System", Microcontrollers and Embedded Systems, vol. 6, (2010).

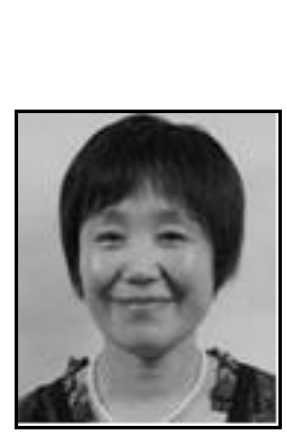

Authors
Li Ma, she received her B.S. degree in 1985 from the Chengdu Institute of Meteorology and her Ph. D degree in 2011 from Nanjing University of Information Scjence and Technology. She is a professor and tutor for graduates in Nanjing Unixersity of Information Science and Technology. Her main research interests include image processing, pattern recognition, and meteorologicalinformation processing and data assimilation.

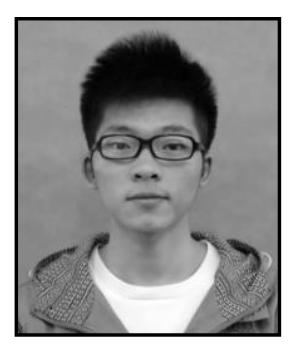

JingZhou Yan, he was born on September 9, 1990. Currently he is a master candidate in Nanjing University of Information Science and Technology. He received his bachelor degree in Nanjing University of Information Science and Technology. His areas of interest are wireless sensor networks, meteorological information processing and data assimilation
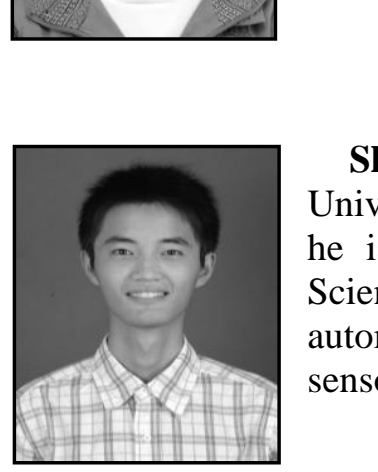

ShuangShuang Yan, he received the bachelor degree in Nanjing University of Information Science and Technology in 2012. Currently he is current master student in Nanjing University of Information Science and Technology. His research interest is focused on the automatic meteorological monitoring which is based on the wireless sensor network.

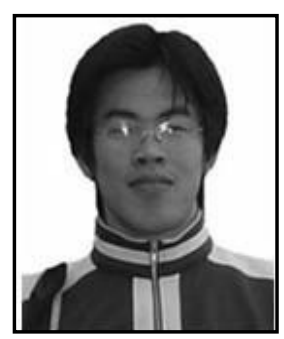

BaoWei Wang, he received his B.S. and Ph.D. degrees in Computer Science from Hunan University in 2005 and 2011, respectively. He is currently working as a lecturer in School of Computer and Software, Nanjing University of Information Science and Technology. His research interests include steganography, wireless networks and securing adhoc networks. 


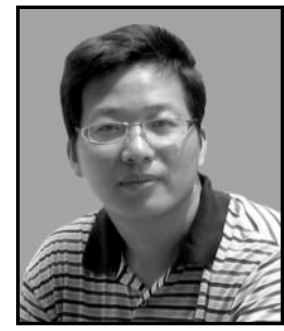

Kuo Liao, he was adf Science degree in 2002. He has worked in the Meteorological Bureau of Hunan Province, Fujian Province amitted to the Department of Atmospheric Sciences, Zhejiang University in 1998 and graduated with a Bachelor ond Ningde City and other departments, mainly engaged in forecasting and business management. Currently he is also a postgraduate in Nanjing University of Information Science and Technology.

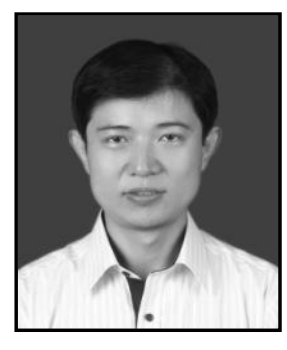

Jin Wang, he received the B.S. and M.S. degree in the Electronical Engineering from Nanjing University of Posts and Telecommunications, China in 2002 and 2005, respectively. He received PhD degree in the Ubiquitous Computing laboratory from the Computer Engineering Department of Kyung Hee University Korea in 2010. Now, he is a professor in the Computer and Sof ware Institute, Nanjing University of Information Science and Techmology. Fis research interests mainly include routing protocol and algorithm, design, performance evaluation and optimization for vireless ad hoc and sensor networks. He is a member of the IEEE and ACM.

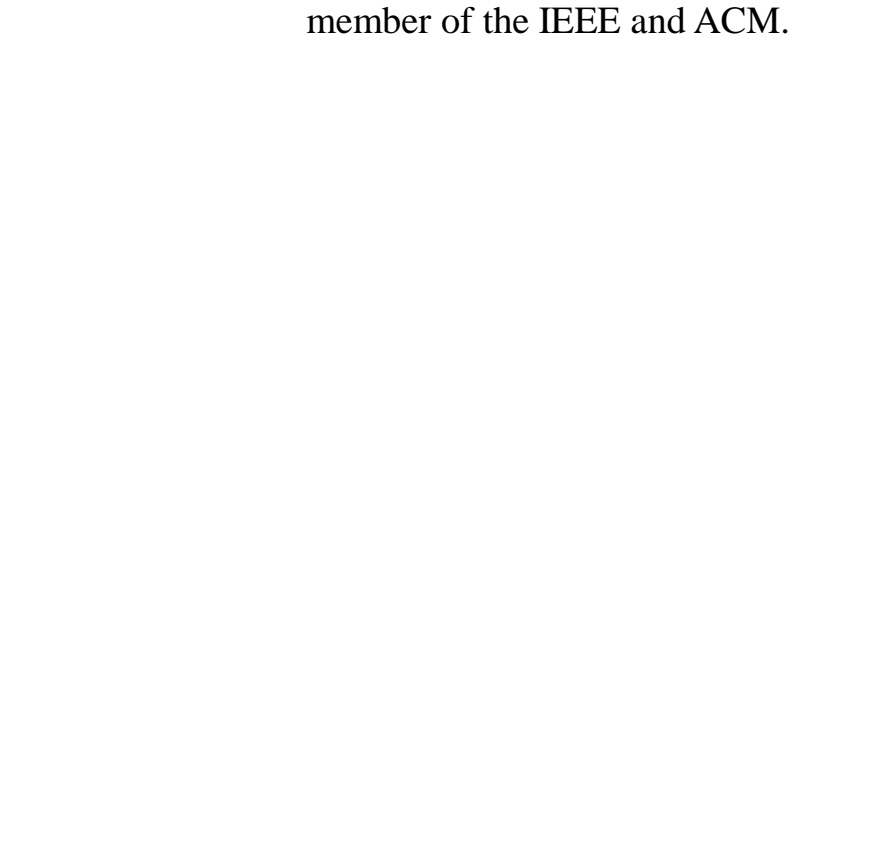


International Journal of Multimedia and Ubiquitous Engineering Vol.9, No.7 (2014)

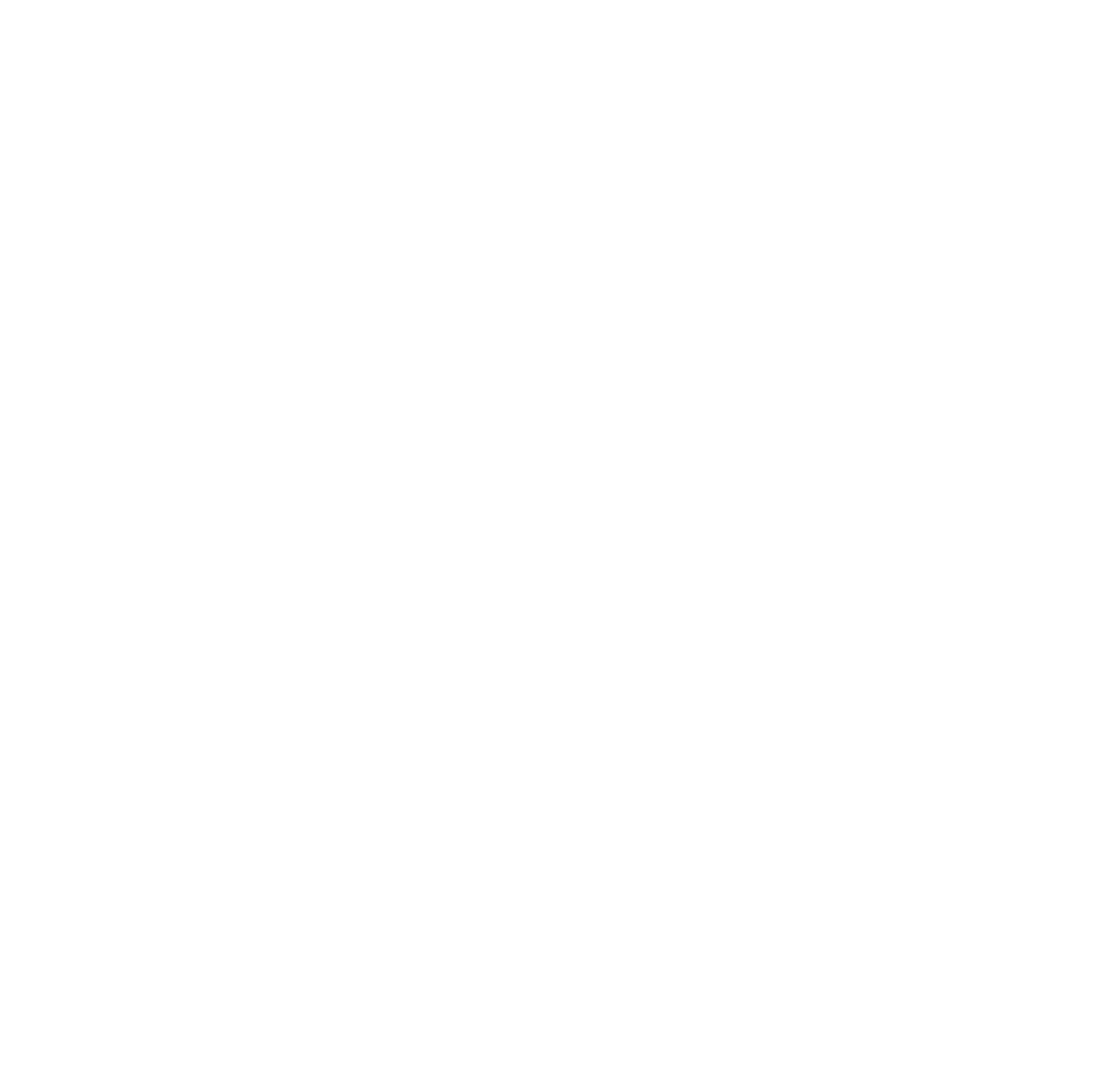

